\title{
Existence of Positive Solutions for an Elastic Beam Equation with Nonlinear Boundary Conditions
}

\author{
Ruikuan Liu and Ruyun Ma \\ Department of Mathematics, Northwest Normal University, Lanzhou 730070, China \\ Correspondence should be addressed to Ruikuan Liu; liuruikuan2008@163.com
}

Received 9 December 2013; Accepted 18 February 2014; Published 20 March 2014

Academic Editor: Ch. Tsitouras

Copyright (C) 2014 R. Liu and R. Ma. This is an open access article distributed under the Creative Commons Attribution License, which permits unrestricted use, distribution, and reproduction in any medium, provided the original work is properly cited.

\begin{abstract}
We study the existence and nonexistence of positive solutions for the following fourth-order two-point boundary value problem subject to nonlinear boundary conditions $u^{\prime \prime \prime \prime}(t)=\lambda f(t, u(t)), t \in(0,1), u(0)=0, u^{\prime}(0)=\mu h(u(0)), u^{\prime \prime}(1)=0, u^{\prime \prime \prime}(1)=$ $\mu g(u(1))$, where $\lambda>0, \mu \geq 0$ are parameters, and $f:[0,1] \times[0,+\infty) \rightarrow(0,+\infty), h:[0,+\infty) \rightarrow[0,+\infty)$, and $g:[0,+\infty) \rightarrow$ $(-\infty, 0]$ are continuous. By using the fixed-point index theory, we prove that the problem has at least one positive solution for $\lambda, \mu$ sufficiently small and has no positive solution for $\lambda$ large enough.
\end{abstract}

\section{Introduction}

In this paper, we study the existence and nonexistence of positive solutions for the following fourth-order boundary value problem with nonlinear boundary conditions

$$
\begin{gathered}
u^{\prime \prime \prime \prime}(t)=\lambda f(t, u(t)), \quad t \in(0,1), \\
u(0)=0, \quad u^{\prime}(0)=\mu h(u(0)), \\
u^{\prime \prime}(1)=0, \quad u^{\prime \prime \prime}(1)=\mu g(u(1)),
\end{gathered}
$$

where $\lambda>0, \mu \geq 0$ are parameters and $f \in C([0,1] \times \mathbb{R}, \mathbb{R})$ and $h, g \in C(\mathbb{R}, \mathbb{R})$ are real functions. If $\lambda=1, \mu=0$, in mechanics, problem (1) is called cantilever beam equation $[1,2]$. The equation describes the deflection of an elastic beam fixed at the left and freed at the right. There are some papers discussing the existence of solutions of the equation by using various methods, such as the lower and upper solution method, the Leray-Schauder continuation method, fixedpoint theory, and the monotone iterative method; see [3-11]. If $\mu \neq 0$, problem (1) has also been studied; see [12-17].

In the case $\mu=1, h(u(0))=0$, Yang et al. [12] obtained sufficient conditions of the existence of two solutions of problem (1) by using variational technique and a three-criticalpoint theorem. Recently, Li and Zhang [13] are concerned with the existence and uniqueness of monotone positive solution of problem (1) with $\lambda=\mu=1, h(u(0))=0$ by using a new fixed-point theorem of generalized concave operators, but some monotone assumptions on $f$ and $g$ are needed.

In 2013, by using a three-critical-point theorem, Cabada and Tersian [14] studied the existence and multiplicity of solutions of problem (1) with $\lambda=\mu, h(u(0))=0$.

A natural question is what would happen if $\lambda \neq \mu$ and $h(u(0)) \neq 0$ ?

Motivated by the above papers, we will prove the existence and nonexistence of positive solution for problem (1) by using the fixed-point index theory with the nonlinearity $f$ satisfying superlinear growth condition at infinity.

We make the following assumptions.

(A1) $f:[0,1] \times[0,+\infty) \rightarrow(0,+\infty)$ is continuous.

$($ A2) $h:[0,+\infty) \rightarrow[0,+\infty)$ is continuous and $g:$ $[0,+\infty) \rightarrow(-\infty, 0]$ is continuous.

(A3) For any $t \in[0,1], f_{\infty}=\lim _{u \rightarrow \infty}(f(t, u) / u)=+\infty$. follows.

The main results of the present paper are summarized as

Theorem 1. Assume that (A1)-(A3) hold. Then problem (1) has at least one positive solution for $\lambda, \mu$ sufficiently small.

Theorem 2. Assume that (A1)-(A3) hold. Then problem (1) has no positive solution for $\lambda$ large enough. 
Remark 3. The results obtained in this paper are not a consequence of the previous theorem in the previous literature. Clearly, the boundary condition of (1) is more general than the above pieces of literature and problem (1) considers two different parameters which is more extensive.

Remark 4. It is pointed out that we do not need any monotone assumption on $f, g$, and $h$, which is weaker than the corresponding assumptions on $f, g$ in [13]. References $[12,14]$ are only considered with the existence of solution for problem (1) with nonlinear boundary condition; however, we study the existence and nonexistence of positive solution of (1).

The remainder of this paper is arranged as follows. Section 2 presents some preliminaries. The proofs of Theorems 1 and 2 are given in Section 3. Finally, we give a simple example to illustrate our main results.

\section{Preliminaries}

In this section we collect some preliminary results that will be used in subsequent sections. Let $X=C[0,1]$; then $X$ is a Banach space under the norm $\|u\|=\max _{t \in[0,1]}|u(t)|$. Define $P=\{u \in X \mid u(t) \geq 0, t \in[0,1]\}$; then $P \subset X$ is a nonnegative cone.

Lemma 5 (see [11, Lemma 2.2]). Let $h \in C[0,1]$; then the solution $u(t)$ of the problem

$$
\begin{gathered}
u^{\prime \prime \prime \prime}(t)=h(t), \quad t \in(0,1), \\
u(0)=u^{\prime}(0)=u^{\prime \prime}(1)=u^{\prime \prime \prime}(1)=0
\end{gathered}
$$

is

$$
u(t)=\int_{0}^{1} G(t, s) h(s) d s
$$

where

$$
G(t, s)= \begin{cases}\frac{1}{6} t^{2}(3 s-t), & 0 \leq t \leq s \leq 1, \\ \frac{1}{6} s^{2}(3 t-s), & 0 \leq s \leq t \leq 1 .\end{cases}
$$

Now, let us set

$$
\begin{array}{r}
\phi(t)=\frac{t^{2}}{2}-\frac{t^{3}}{6}, \quad \Phi(t)=h(u(0)) t-g(u(1)) \phi(t), \\
\forall t \in[0,1] .
\end{array}
$$

It follows from (3) and (5) with simple computation that problem (1) is equivalent to integral equation

$$
u(t)=\lambda \int_{0}^{1} G(t, s) f(s, u(s)) d s+\mu \Phi(t):=T u(t) .
$$

Note that for any $u \in X$ the function $(T u)(t)$ satisfies the boundary conditions in (1) by the definition of Green's function $G(t, s)$. In view of Lemma 5 , it is easy to see that $u \in X$ is a fixed point of the operator $T$ if and only if $u \in X$ is a solution of problem (1).
Lemma 6 (see [6, Lemma 2.2], [13, Lemma 2.1]). $G(t, s), \phi(t)$ have the following properties:

(i) $G(t, s)>0, \forall 0<t, s<1$;

(ii) $\max _{t \in[0,1]} G(t, s) \leq G(1, s)=(1 / 6) s^{2}(3-s), \forall 0 \leq s \leq 1$;

(iii) $G(t, s) \geq t^{2} G(1, s) \geq\left(t^{2} / 3\right) G(1, s), \forall 0 \leq t, s \leq 1$;

(iv) $t^{2} / 3 \leq \phi(t) \leq t^{2} / 2, \forall 0 \leq t \leq 1$.

Lemma 7. $\Phi(t)$ has the following properties:

(i) $\Phi(t) \leq \Phi(1) \leq h(u(0))-g(u(1)), \forall 0<t<1$;

(ii) $\Phi(t) \geq \Phi\left(t^{2} / 3\right) \geq 0, \forall 0<t<1$.

Proof. First, we will prove (i). For any $t \in[0,1]$, it follows that

$$
\Phi^{\prime}(t)=h(u(0))-g(u(1))\left(t-\frac{1}{2} t^{2}\right) .
$$

From (A2), $\Phi^{\prime}(t) \geq 0$. So $\Phi(t)$ is nondecreasing for $t \in[0,1]$. Moreover,

$$
\Phi(t) \leq \Phi(1)=h(u(0))-\frac{1}{3} g(u(1)) \leq h(u(0))-g(u(1)),
$$

so (i) holds.

Now, we prove (ii). For any $t \in[0,1]$, obviously, $t \geq t^{2} / 3 \geq$ 0 .

From (i), $\Phi(t)$ is nondecreasing for $t \in[0,1]$; then $\Phi(t) \geq$ $\Phi\left(t^{2} / 3\right) \geq \Phi(0)=0$.

For any fixed $\tau \in(0,1)$, define the cone

$$
K=\left\{u \in P \mid \min _{t \in[\tau, 1]} u(t) \geq \frac{\tau^{2}}{3}\|u\|\right\} .
$$

Then $K \subset X$ is a positive cone; letting $K_{r}=\{u \in K \mid\|u\|<r\}$, $\partial K_{r}=\{u \in K \mid\|u\|=r\}$ with $r>0$ is a constant.

Lemma 8. Assume that (A1) and (A2) hold; then $T: K \rightarrow K$ is completely continuous.

Proof. Obviously, for any $t \in[0,1], u \in K$, it follows that $T u(t) \geq 0$.

For any $u \in K$, from (6) and Lemma $7(\mathrm{i})$, we have

$$
\begin{aligned}
\max _{t \in[0,1]} T(u(t))= & \max _{t \in[0,1]}\left(\lambda \int_{0}^{1} G(t, s) f(s, u(s)) d s+\mu \Phi(t)\right) \\
\leq & \lambda \int_{0}^{1} G(1, s) f(s, u(s)) d s \\
& +\mu(h(u(0))-g(u(1))) .
\end{aligned}
$$

Hence

$\|T u\| \leq \lambda \int_{0}^{1} G(1, s) f(s, u(s)) d s+\mu(h(u(0))-g(u(1)))$. 
For any fixed $\tau \in(0,1)$, (11) together with Lemma 7(ii), we get

$$
\begin{aligned}
\min _{\tau \leq t \leq 1} T(u(t))= & \min _{\tau \leq t \leq 1}\left(\lambda \int_{0}^{1} G(t, s) f(s, u(s)) d s\right. \\
& +\mu(h(u(0)) t-g(u(1)) \phi(t))) \\
\geq & \frac{\tau^{2}}{3}\left(\lambda \int_{0}^{1} G(1, s) f(s, u(s)) d s\right. \\
& +\mu(h(u(0))-g(u(1)))) \\
\geq & \frac{\tau^{2}}{3}\|T u\| .
\end{aligned}
$$

So, $T(K) \subset K$.

According to (A1), (A2), and Arzela-Ascoli theorem, it is not difficult to verify that $T: K \rightarrow K$ is completely continuous.

The following well-known fixed-point index theorem in cones is crucial to our arguments.

Lemma 9 (see [18]). Let $E$ be a Banach space and let $K \subset E$ be a cone. For $r>0$, define $K_{r}=\{u \in K \mid\|u\|<r\}$. Assume that $T: \bar{K}_{r} \rightarrow K$ is compact map such that $T u \neq u$ for $u \in \partial K_{r}=$ $\{u \in K \mid\|u\|=r\}$.

(i) If $\|T u\| \geq\|u\|$ for $u \in \partial K_{r}$, then $i\left(T, K_{r}, K\right)=0$.

(ii) If $\|T u\| \leq\|u\|$ for $u \in \partial K_{r}$, then $i\left(T, K_{r}, K\right)=1$.

\section{Proof of Main Results}

In this section, we will prove our main results.

Proof of Theorem 1. Let

$$
M=\max _{(t, s) \in[0,1] \times[0,1]} G(t, s)>0 .
$$

Let $q>0$; set

$$
I(q)=M \max _{u \in K,\|u\|=q}\left(\int_{0}^{1} f(s, u(s)) d s\right)>0 .
$$

For any number $r_{1}>0$, let $K_{r_{1}}=\left\{u \in K \mid\|u\| \leq r_{1}\right\}$. Choose $\sigma>0$ so that

$$
\begin{gathered}
\sigma \leq \frac{r_{1}}{2 I\left(r_{1}\right)}, \quad \forall u \in \partial K_{r_{1}}, \\
\sigma(h(u(0))-g(u(1))) \leq \frac{r_{1}}{2}, \quad \forall u \in \partial K_{r_{1}} .
\end{gathered}
$$

Then, for $\lambda \leq \sigma, \mu \leq \sigma$ and $u \in \partial K_{r_{1}}$, combining (6) with Lemma 7 , we have

$$
\begin{aligned}
T u(t) \leq & \lambda M \int_{0}^{1} f(s, u(s)) d s \\
& +\mu(h(u(0))-g(u(1))) \\
\leq & \sigma M \int_{0}^{1} f(s, u(s)) d s+\sigma(h(u(0))-g(u(1))) \\
\leq & \sigma I\left(r_{1}\right)+\frac{r_{1}}{2} \leq r_{1},
\end{aligned}
$$

which implies that

$$
\|T u\| \leq r_{1}=\|u\|, \quad u \in \partial K_{r_{1}} .
$$

Thus, Lemma 9 implies that

$$
i\left(T, K_{r_{1}}, K\right)=1 .
$$

According to (A3), for any $t \in[0,1]$, there exists a $p>0$ such that

$$
f(t, u) \geq \eta u, \quad \forall u \geq p,
$$

where $\eta$ is chosen so that

$$
\lambda\left(\frac{\tau^{2}}{3}\right)^{2} \eta \int_{\tau}^{1} G(1, s) d s \geq 1, \quad \forall \tau \in(0,1) .
$$

For any $\tau \in(0,1)$, choose $r_{2}>\max \left\{\left(3 / \tau^{2}\right) p, 2 r_{1}\right\}$, and set $K_{r_{2}}=\left\{u \in K \mid\|u\| \leq r_{2}\right\}$. If $u \in \partial K_{r_{2}}$, then

$$
\min _{\tau \leq t \leq 1} u(t) \geq \frac{\tau^{2}}{3} r_{2}>p
$$

It follows from (A2) and Lemma 6 that

$$
\begin{aligned}
\min _{t \in[\tau, 1]} T(u(t)) & \geq \min _{t \in[\tau, 1]}\left(\lambda \int_{0}^{1} G(t, s) f(s, u(s)) d s\right) \\
& \geq \lambda \frac{\tau^{2}}{3} \int_{0}^{1} G(1, s) f(s, u(s)) d s \\
& \geq \lambda \frac{\tau^{2}}{3} \int_{0}^{1} G(1, s) \eta u(s) d s \\
& \geq \lambda \frac{\tau^{2}}{3} \eta \int_{\tau}^{1} G(1, s) u(s) d s \\
& \geq \lambda\left(\frac{\tau^{2}}{3}\right)^{2} \eta \int_{\tau}^{1} G(1, s) d s\|u\| \\
& \geq\|u\|,
\end{aligned}
$$

which implies that

$$
\|T(u)\| \geq\|u\|, \quad u \in \partial K_{r_{2}} .
$$


Hence, from Lemma 9, we get

$$
i\left(T, K_{r_{2}}, K\right)=0 \text {. }
$$

From (18) and (24), it follows that

$$
i\left(T, K_{r_{2}} \backslash \bar{K}_{r_{1}}, K\right)=i\left(T, K_{r_{2}}, K\right)-i\left(T, K_{r_{1}}, K\right)=-1 .
$$

Therefore, $T$ has a fixed point in $K_{r_{2}} \backslash \bar{K}_{r_{1}}$; that is, problem (1) has at least one positive solution.

Proof of Theorem 2. From (A1) and (A3), for any $t \in[0,1]$, there is a constant $c$ such that

$$
f(t, u) \geq c u, \quad \forall u \geq 0 .
$$

Let $u \in X$ be a positive solution of (1); then $u \in K$ satisfies (6). Choose $\lambda$ large enough such that

$$
\lambda\left(\frac{\tau^{2}}{3}\right)^{2} c \int_{\tau}^{1} G(1, s) d s>1, \quad \forall \tau \in(0,1) .
$$

For any $\tau \in(0,1)$, we have

$$
\begin{aligned}
\min _{t \in[\tau, 1]} u(t) & \geq \min _{t \in[\tau, 1]}\left(\lambda \int_{0}^{1} G(t, s) f(s, u(s)) d s\right) \\
& \geq \lambda \frac{\tau^{2}}{3} \int_{\tau}^{1} G(1, s) c u(s) d s \\
& \geq \lambda\left(\frac{\tau^{2}}{3}\right)^{2} c \int_{\tau}^{1} G(1, s) d s\|u\| \\
& >\|u\|,
\end{aligned}
$$

which is a contradiction. Therefore, problem (1) has no positive solution for $\lambda$ large enough.

Finally, we give an example to illustrate our main result.

Example 10. Consider the following fourth-order two-point problem with nonlinear boundary conditions:

$$
\begin{gathered}
u^{\prime \prime \prime \prime}(t)=\lambda\left(u^{3}(t)(3+\sin (u(t)))+t^{2}+1\right), \quad t \in(0,1), \\
u(0)=0, \quad u^{\prime}(0)=\mu\left(2[u(0)]^{2}+1\right), \\
u^{\prime \prime}(1)=0, \quad u^{\prime \prime \prime}(1)=\mu\left([-(u(1))]^{1 / 2}-1\right) .
\end{gathered}
$$

Clearly, the nonlinearity

$$
\begin{gathered}
f(t, u)=u^{3}(t)(3+\sin (u(t)))+t^{2}+1, \\
(t, u) \in[0,1] \times[0, \infty), \\
h(s)=2 s^{2}+1, \quad g(s)=-s^{1 / 2}-1, \quad \forall s \in[0,+\infty) .
\end{gathered}
$$

It is easy to check that (A1)-(A3) are satisfied. By simple computation, we have $M=1 / 2$. Set $q=2$; then $I(q)=17$.
If $r_{1}=4$ (where $r_{1}$ can be any real number greater than 0 ), $u \in \partial K_{r_{1}}$, then $r_{1} / 2 I(q)=2 / 17, \Phi(t) \leq 36$, and take $\sigma=1 / 20$; then $\|T(u)\| \leq\|u\|$ obviously holds for $\lambda, \mu<1 / 20$.

Choosing $r_{2}=9$, it is easy to verify that

$$
\|T(u)\| \geq\|u\|, \quad \forall u \in \partial K_{r_{2}} .
$$

From Theorem 1, T has a fixed point in $K_{r_{2}} \backslash \bar{K}_{r_{1}}$; that is, problem (29) has at least one positive solution.

For any $t \in[0,1]$, there is a constant $c=3$, so that

$$
f(t, u) \geq 3 u, \quad \forall u \geq 0 .
$$

If $\tau=1 / 2$, then problem (29) has no positive solution for $\lambda>450$.

\section{Conflict of Interests}

The authors declare that there is no conflict of interests regarding the publication of this paper.

\section{Acknowledgments}

The authors are very grateful to the anonymous referees for their valuable suggestions. This work was supported by NSFC (nos. 11361054 and 11201378), SRFDP (no. 2012 6203110004), and Gansu Provincial National Science Foundation of China (no. 1208RJZA258).

\section{References}

[1] R. P. Agarwal, Boundary Value Problems for Higher Order Differential Equations, World Scientific Publishing, Singapore, 1986.

[2] A. R. Aftabizadeh, "Existence and uniqueness theorems for fourth-order boundary value problems," Journal of Mathematical Analysis and Applications, vol. 116, no. 2, pp. 415-426, 1986.

[3] R. P. Agarwal, "On fourth order boundary value problems arising in beam analysis," Differential and Integral Equations, vol. 2, no. 1, pp. 91-110, 1989.

[4] J. R. Graef and B. Yang, "Existence and nonexistence of positive solutions of fourth order nonlinear boundary value problems," Applicable Analysis, vol. 74, no. 1-2, pp. 201-214, 2000.

[5] R. Ma and H. Wang, "On the existence of positive solutions of fourth-order ordinary differential equations," Applicable Analysis, vol. 59, no. 1-4, pp. 225-231, 1995.

[6] Q. Yao, "Positive solutions of nonlinear beam equations with time and space singularities," Journal of Mathematical Analysis and Applications, vol. 374, no. 2, pp. 681-692, 2011.

[7] Q. Yao, "Monotonically iterative method of nonlinear cantilever beam equations," Applied Mathematics and Computation, vol. 205, no. 1, pp. 432-437, 2008.

[8] A. Cabada, J. Cid, and L. Sanchez, "Positivity and lower and upper solutions for fourth order boundary value problems," Nonlinear Analysis: Theory, Methods \& Applications, vol. 67, no. 5, pp. 1599-1612, 2007.

[9] Z. Bai, "The upper and lower solution method for some fourthorder boundary value problems," Nonlinear Analysis: Theory, Methods \& Applications, vol. 67, no. 6, pp. 1704-1709, 2007.

[10] Z. Bai, B. Huang, and W. Ge, "The iterative solutions for some fourth-order p-Laplace equation boundary value problems," Applied Mathematics Letters, vol. 19, no. 1, pp. 8-14, 2006. 
[11] Q. Yao, "Local existence of multiple positive solutions to a singular cantilever beam equation," Journal of Mathematical Analysis and Applications, vol. 363, no. 1, pp. 138-154, 2010.

[12] L. Yang, H. Chen, and X. Yang, "The multiplicity of solutions for fourth-order equations generated from a boundary condition," Applied Mathematics Letters, vol. 24, no. 9, pp. 1599-1603, 2011.

[13] S. Li and X. Zhang, "Existence and uniqueness of monotone positive solutions for an elastic beam equation with nonlinear boundary conditions," Computers \& Mathematics with Applications, vol. 63, no. 9, pp. 1355-1360, 2012.

[14] A. Cabada and S. Tersian, "Multiplicity of solutions of a two point boundary value problem for a fourth-order equation," Applied Mathematics and Computation, vol. 219, no. 10, pp. 52615267, 2013.

[15] E. Alves, T. F. Ma, and M. L. Pelicer, "Monotone positive solutions for a fourth order equation with nonlinear boundary conditions," Nonlinear Analysis: Theory, Methods \& Applications, vol. 71, no. 9, pp. 3834-3841, 2009.

[16] T. F. Ma, "Positive solutions for a beam equation on a nonlinear elastic foundation," Mathematical and Computer Modelling, vol. 39, no. 11-12, pp. 1195-1201, 2004.

[17] B. Yang, "Positive solutions for the beam equation under certain boundary conditions," Electronic Journal of Differential Equations, vol. 78, pp. 1-8, 2005.

[18] D. J. Guo and V. Lakshmikantham, Nonlinear Problems in Abstract Cones, Academic Press, New York, NY, USA, 1988. 


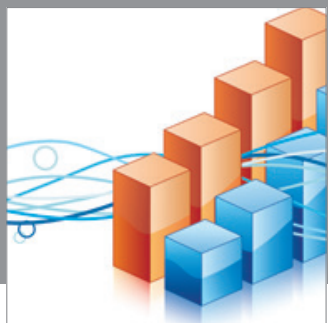

Advances in

Operations Research

mansans

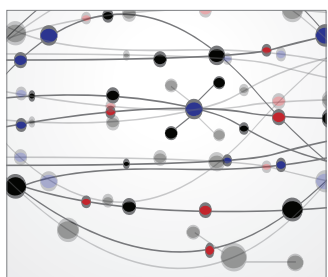

The Scientific World Journal
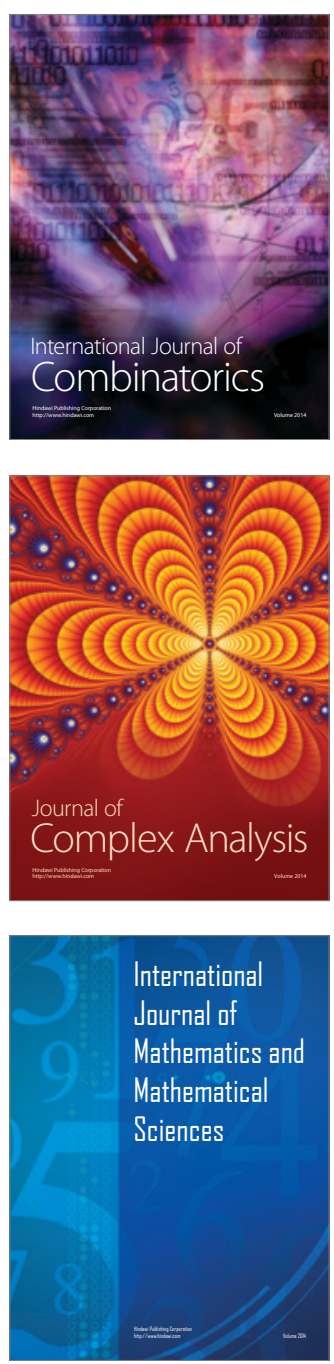
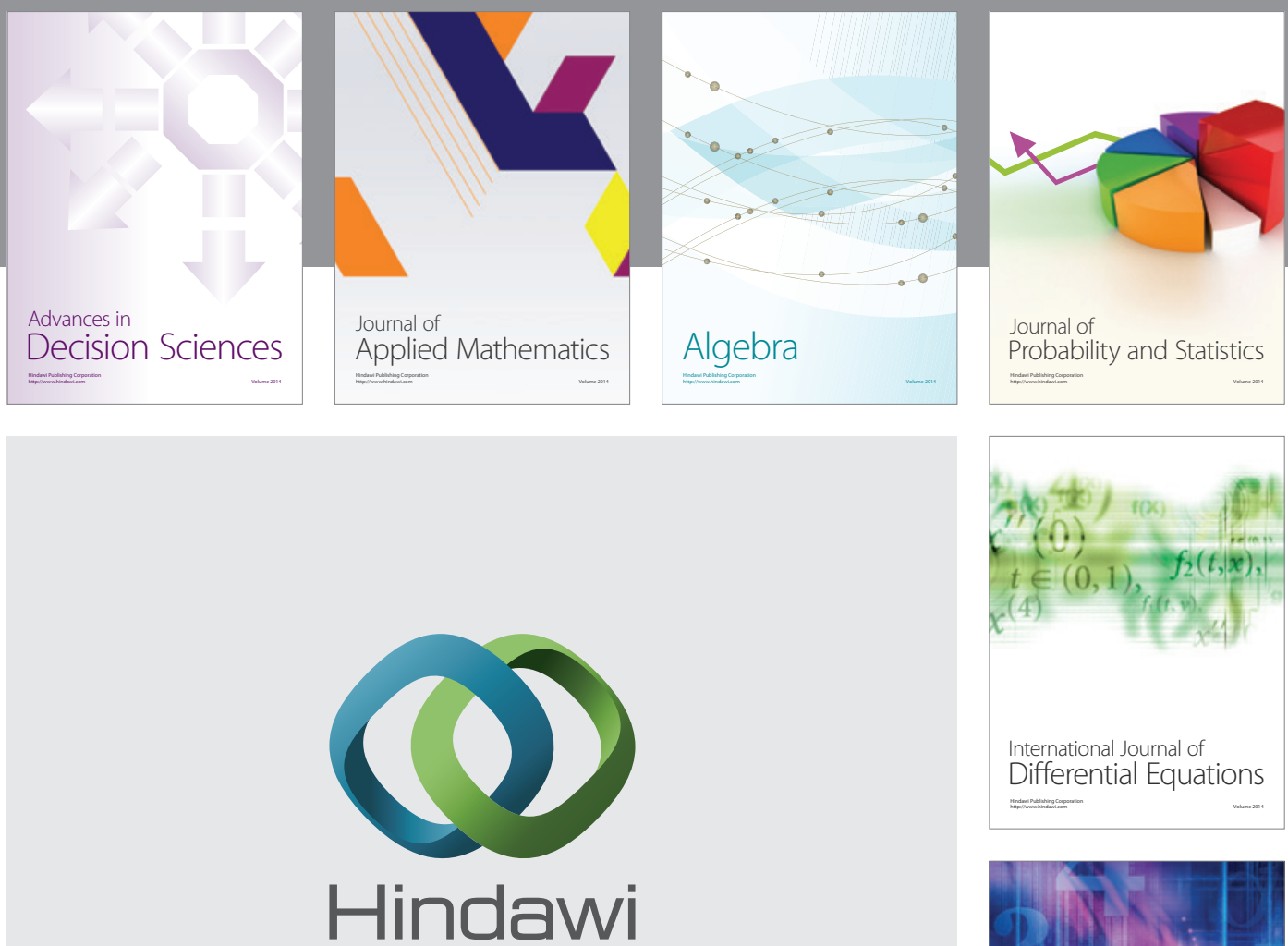

Submit your manuscripts at http://www.hindawi.com
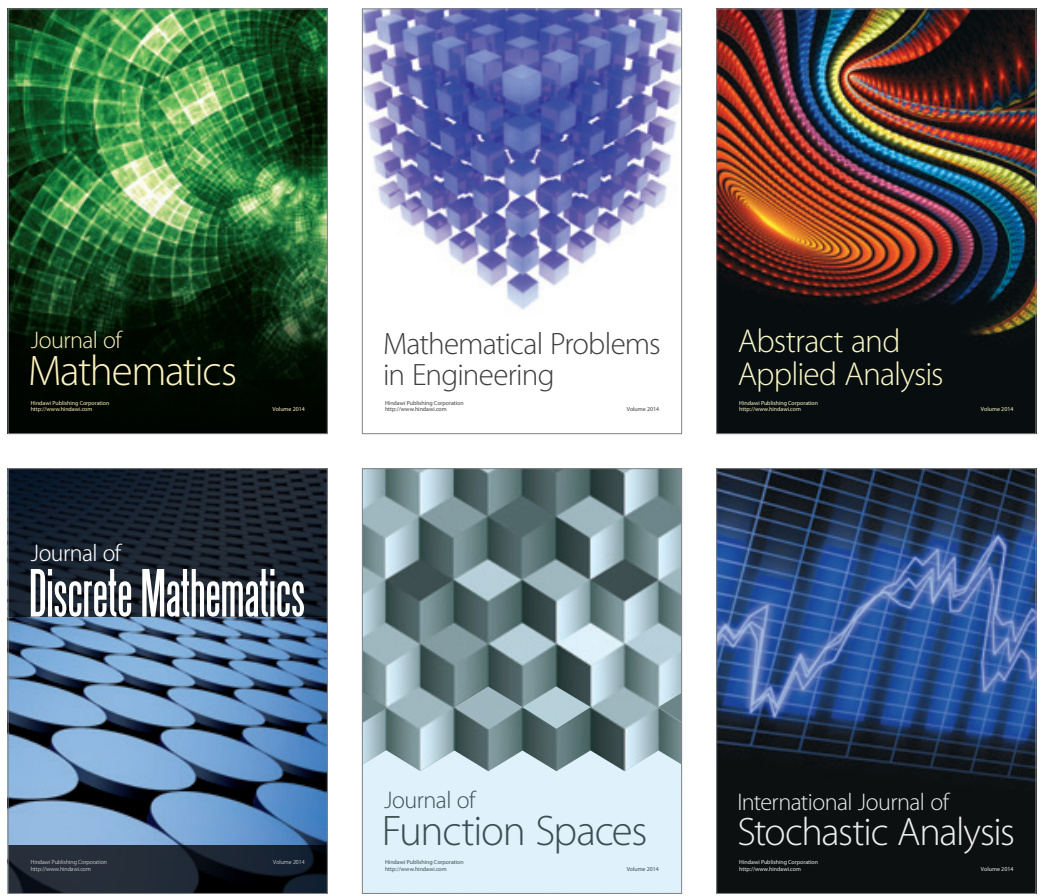

Journal of

Function Spaces

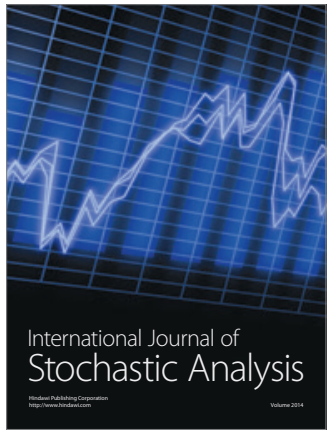

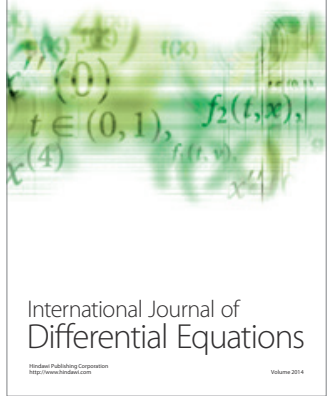
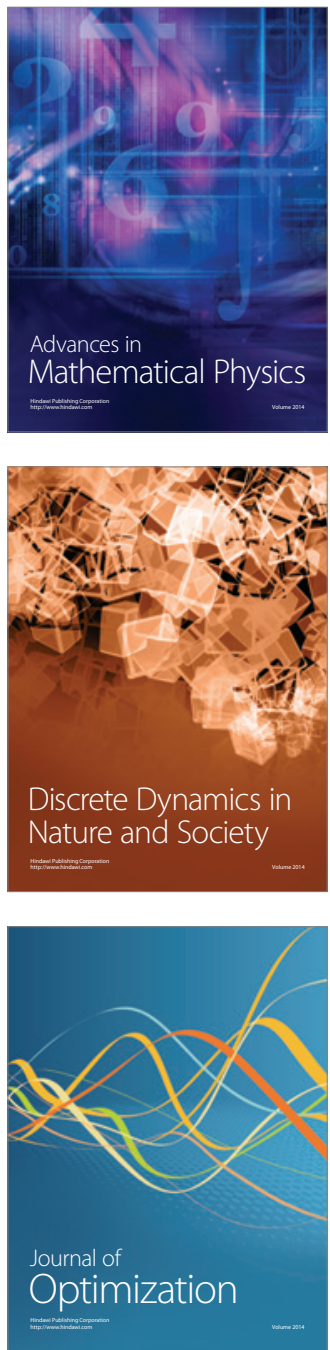